CARR, BRENDAN M., M.S. Examining the Time Course of Attention in a Soccer Kick Using a Dual Task Paradigm. (2010)

Directed by Dr. Jennifer L. Etnier. 50 pp.

A dual-task paradigm was implemented to determine the time course of attention demands of a soccer penalty kick. A sample of experienced soccer players was selected to participate in the study. Participants $(\mathrm{N}=15)$ were asked to perform a 12-yard soccer-style penalty kick into a FIFA-regulation soccer goal that was divided into smaller scoring zones. As part of the dual task paradigm, participants were instructed to respond to an audible cue that was administered during one of three probe positions (PP) during the primary task. Tests were performed with both the dominant foot (DFP) and the non-dominant foot (NDFP). The order of presentation of the tasks and of the probe positions was randomized and counterbalanced. I hypothesized that the Verbal Response Reaction Time (VRRT) would follow the pattern observed in previous research conducted with other sport skills. Reaction times would be slowest at the beginning and end of the performance of the motor skill in both the DFP and NDFP situations. I also hypothesized that overall VRRT would be slower in the NDFP than DFP, but that kicking foot would not affect the pattern of attentional demands. Results indicated that VRRT was slowest at PP1 for both the DFP and the NDFP and that VRRT was significantly slower at PP1 for the DFP than for the NDFP. This suggests that soccer players may engage in more complex planning during the preparatory phases when executing a kick with their dominant foot or, alternatively, that the determinant of attentional demands is the support foot rather than the kicking foot. Future research should be designed to further our understanding of foot dominance with regard to kicking and to explore attentional demands of striking tasks. 


\title{
EXAMINING THE TIME COURSE OF ATTENTION IN A SOCCER KICK USING A DUAL TASK PARADIGM
}

\author{
by \\ Brendan M. Carr

\begin{abstract}
A Thesis Submitted to
the Faculty of The Graduate School at

The University of North Carolina at Greensboro

in Partial Fulfillment

of the Requirements for the Degree

Master of Science
\end{abstract}

\section{Greensboro}

2010

Approved by

Committee Chair 
To my parents, for all their unconditional love and support. 


\begin{abstract}
APPROVAL PAGE
This thesis has been approved by the following committee of the Faculty of The Graduate School at The University of North Carolina at Greensboro.
\end{abstract}

Committee Chair

Committee Members

Date of Acceptance by Committee

Date of Final Oral Examination 


\section{ACKNOWLEDGMENTS}

Many thanks to Dr. Jennifer Etnier for all her patience, guidance, and support throughout this project and my graduate career.

Thank you to my thesis committee, Dr. Bob Christina and Dr. Kurt Kornatz. I appreciate your wisdom and guidance.

Also a special thanks to Marc Nicholls and the parents and players of the Greensboro Youth Soccer Club for their participation and assistance in recruiting subjects. 


\section{TABLE OF CONTENTS}

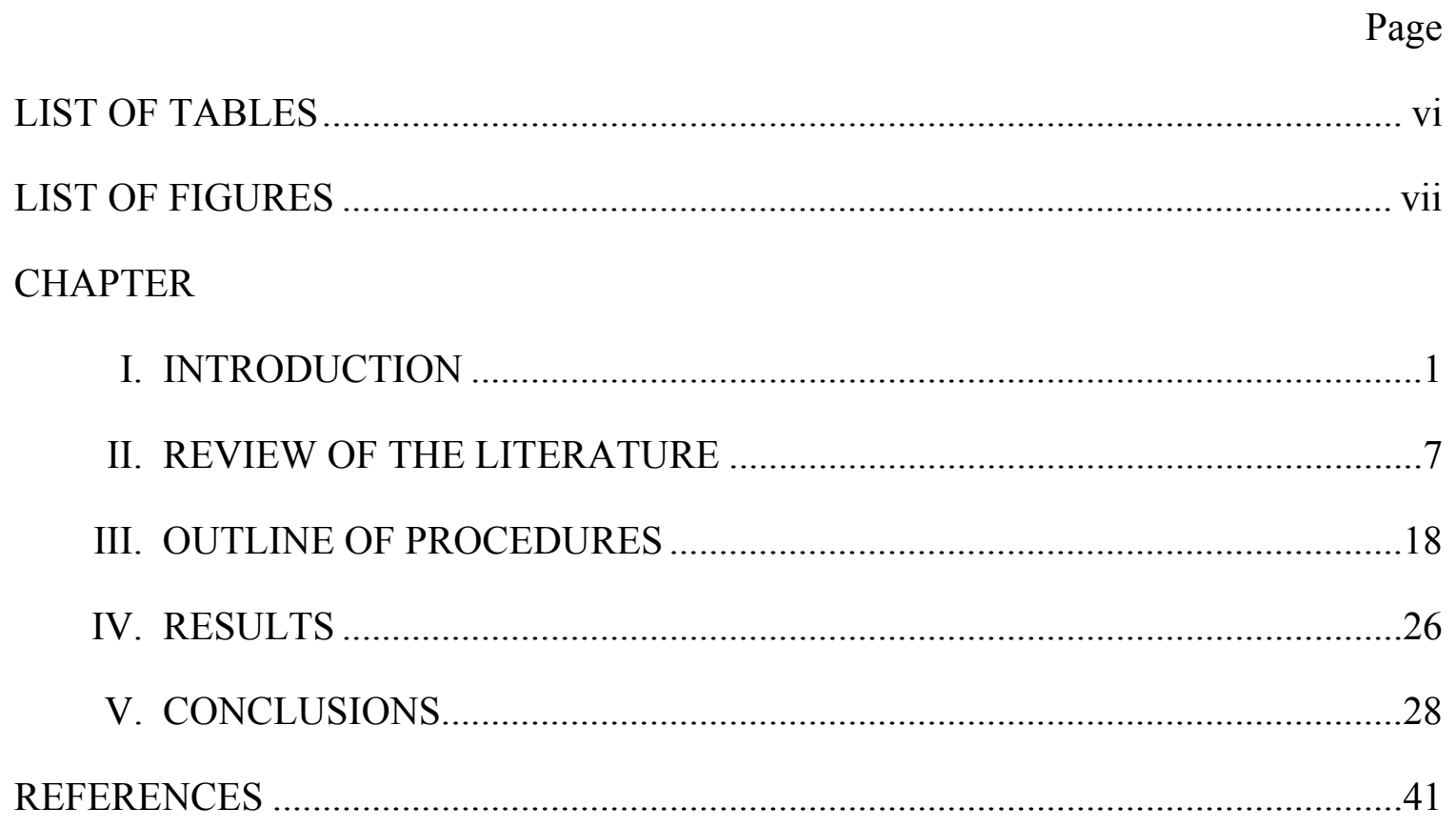




\section{LIST OF TABLES}

Page

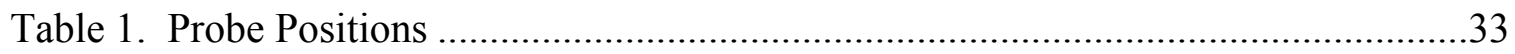

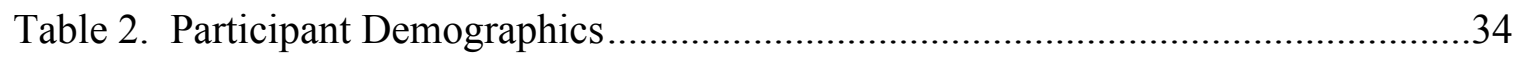

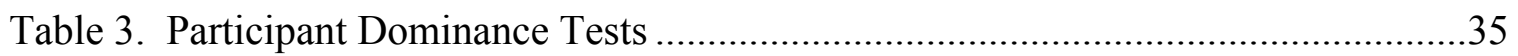

Table 4. Influence of Condition on Kick Performance........................................................36

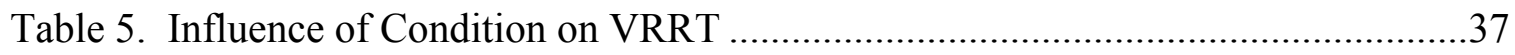




\section{LIST OF FIGURES}

Figure 1. Soccer Kick Task Design .38

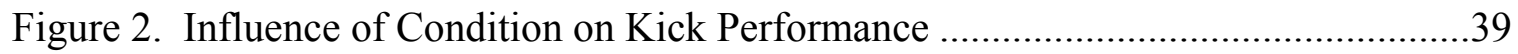

Figure 3. Influence of Condition on VRRT .40 


\section{CHAPTER I}

\section{INTRODUCTION}

In what is still considered the most comprehensive explanation, William James (1890, pp. 403-404) defined attention as:

It is the taking possession by the mind, in clear and vivid form, of one out of what seem several simultaneously possible objects or trains of thought. Focalization, concentration, of consciousness are of its essence. It implies withdrawal from some things in order to deal effectively with others, and is a condition which has a real opposite in the confused, dazed, scatterbrained state which in French is called distraction, and Zerstreutheit in German.

This definition assumes volitional control over when and what we focus our attention on.

We are frequently under a barrage of multiple streams of information and must select where to direct our attentional capacities. James' classic definition also infers that multitasking may require a prioritization of tasks for successful completion of one or both tasks.

These assumptions have formed the capacity theory of attention (Kahneman, 1973). At some point, the cognitive demands of performing multiple tasks exceed attentional capacities and performance decrements appear on one or both tasks. In a sense, the human brain operates similarly to a computer processor. Whether equated to information processing space, total capacity, or 
available cognitive reserve; attention is a limited resource (Singer, Hausenblas, and Janelle, 2001). If the demands of simultaneously executing multiple "programs" exceed available resources, overall performance slows, stagnates, or results in system failure.

A dual-task paradigm is used in research to measure the attentional demands of a specific task (Kahneman, 1973). After providing baseline performance measures, individuals are asked to complete two concurrent tasks and their ability to divide attention between those tasks is assessed. If performance diminishes on a relatively simple, concurrent secondary task, it is inferred that the primary task required a majority of the individual's cognitive resources and that there were not enough resources remaining to complete the secondary task successfully. If there is little or no decline in the performance of the secondary task, it is implied that the primary task did not require substantial attention and there was sufficient residual processing capacity for both tasks. If conducted properly, the dual-task paradigm can be used to accurately quantify attentional demands.

Dual-task techniques are frequently used in establishing the time course of attention in motor skills (Singer, Hausenblas, \& Janelle, 2001). Castiello and Umilta (1988) looked at a serve-receive task in volleyball, 100-meter sprint, 110-meter hurdles, and a serve-return task in tennis. In the volleyball serve-receive task and serve-return task in tennis, reaction times were fastest at the point of contact by the server. During the volleyball task, reaction times were slower as the ball carried over the net and slowest when the receiving player was about to hit the ball. During the tennis serve-return, reaction times were slowest as the ball bounced in the near court then were faster as the 
receiving player initiated the return. For both sprint events, reaction times were slower at the beginning and end of each race than at the intermediate time points. Rose and Christina (1990) extended this line of applied research by examining the distribution of attention during the aiming phase of pistol shooting performed by expert and novice shooters. The study used a secondary task that required participants to depress a microswitch with their non-shooting hand as quickly as possible in response to an auditory tone delivered randomly at one of six predetermined time points after the shooter had raised the pistol to the "hold" or "ready" position. Across both skill levels, level of attention increased linearly over time as a function of the presentation of the secondary task.

Prezuhy and Etnier (2001) used a dual-task paradigm to assess attention in forty competitive horseshoe pitchers under normal and an experimental "difficult" condition. The secondary task was a reaction time test to an auditory cue presented at one of three probe positions during the toss. Reaction times were greatest at the beginning and end of the tossing motion under both levels of difficulty. Following that, Sibley and Etnier (2004) employed the dual-task paradigm to observe the relationship between decisionmaking and the time course of attentional demands during a volleyball set. Results indicated that the highest attentional demands were evident when the ball was initially tossed towards the participant. The initial tracking of the ball's flight pattern, choosing the direction of the set, and planning the subsequent motor response happen quickly and require greater cognitive resources for a successful pass to occur. Attentional demands were less during the ball's flight. The second most attention-demanding portion of the volleyball set occurred when the athlete was just making contact with the volleyball. 
In a recent study conducted by Price et al. (2009), the pattern of attention demands during the closed-skill task of a basketball free throw were examined. Results indicated that attentional demands were greatest during the pre-shot routine and as the participant was preparing to shoot. Attentional demands as the shot was actually being made and during ball flight were not significantly different than baseline. The results of these studies support a trend toward higher demands at the beginning and end of a movement for certain types of gross motor tasks (Prezuhy \& Etnier, 2001). As presented in Prezuhy and Etnier (2001), past research suggested attentional patterns for fine motor skills will follow a linear pattern and gross motor sport skills will follow a non-linear pattern. However, newer findings suggest that the division between fine and gross motor skills may be too general. Prezuhy and Etnier (2001) suggested that it may be best to categorize tasks based on their required interactions with external stimuli. Gross motor skills such as horseshoe pitching or throwing could be classified as "projection" tasks; receiving a tennis or volleyball serve could be classified as a "reception" task; kicking a football through the uprights or hitting a golf ball could be considered a "striking" task. Fine motor skills such as pistol shooting could be classified as "aiming” tasks.

While applied research is limited, several key studies have shown that the dualtask paradigm and Verbal Response Reaction Time (VRRT) probe technique are effective means for measuring time-course attentional demands in performing sportrelated motor skills. Attention research relevant to soccer has focused on continuous tasks (ie. dribbling) rather than discrete tasks (ie. single kick). This study presents a novel task for attentional research. The soccer kick falls under the proposed "striking" category of motor skill which is a category of motor skills in which the dual-task 
paradigm has not yet been applied. Thus, this study will extend our understanding of attentional demands of sport skills by focusing on a striking task that requires the participant to use her foot to strike a ball for distance and accuracy. Inclusion of the Non-Dominant Foot Position (NDFP) also adds to the literature by addressing foot dominance as a degree of difficulty.

\section{Purpose and Hypotheses}

As the name implies, the "penalty kick" is a soccer task used during games to assess a penalty occurring inside the goal area for a rules infraction resulting in a direct kick. It can also be used as a tie-breaker during competition at the end of regulation time and subsequent overtime periods where the match has resulted in a stalemate. During the penalty kick, the soccer ball is placed central to the mouth of the goal and 12-meters away from the goal line.

Under normal game conditions, a goalkeeper would be situated between the player taking the kick and the goal to prevent the opposing team from scoring. Given the position of the goalkeeper, the shot location with the highest likelihood of scoring would be just inside the side frames outside the reach of the goalkeeper's outstretched hands. This task requires accurate planning and timing by the player kicking the ball. For successful completion of this task, attentional skills and physical ability are required.

The purpose of this study is to examine the attentional demands of a soccer penalty kick. Kick accuracy is the primary outcome measure for this experiment. Verbal response reaction time (VRRT) to the auditory stimuli is the secondary task. During time points where the attentional demands of the primary task are greatest, concurrent 
administration of the auditory stimuli should tax the participant's attentional capacity most and result in slower VRRT's. Although striking tasks have not yet been studied using a dual-task paradigm, based upon findings with tasks that have been identified as projection tasks, I hypothesize that the VRRT's will be slowest at the start and end of the movement. Execution of the gross motor program is believed to be an automatic process, therefore the initial planning and adjustment phases are considered the most "attentionintensive" and vulnerable to distraction. In the second experimental situation, I believe non-dominant foot performance (NDFP) will follow the same pattern as dominant foot performance (DFP). It is also hypothesized that VRRT will be slower in the NDFP than DFP, but that the attentional pattern will not differ as a function of this manipulation of task difficulty. 


\section{CHAPTER II}

\section{REVIEW OF THE LITERATURE}

\section{Attentional Demands in Sport}

Abernethy, Summers, and Ford (1998) caution researchers against four major methodological concerns when conducting research using a dual-task paradigm. The first major concern involves the selection of secondary tasks. It is imperative that the secondary task not create structural interference with the first task. Structural interference occurs when two concurrent tasks require the use of the same perceptual or response production systems. The secondary task should produce capacity interference, or the challenging of the individual's general attentional capacity. Simple reaction time (RT) tests are often used to generate capacity interference.

The second major methodological concern is the presentation of the secondary task stimulus. Individuals may attempt to anticipate the secondary task stimulus thereby shifting primary attention to the secondary task. Random presentation of the secondary task stimulus and the use of catch trials, where no secondary task stimulus is presented, can help avoid this expectancy bias.

To ensure testing validity, it is important to establish baseline performance measures of both the primary and secondary task independently of each other. Comparisons made between primary task performance and dual task performance provide feedback as to whether the participant maintained major focus on the primary task. 
Comparisons made between secondary task performance and dual task performance allow analysis of the attentional demands required for the primary task performance.

Castiello and Umilta (1988) conducted a groundbreaking study to measure the time course of attentional demands of four athletic events in real world settings. They looked at a serve-receive task in volleyball, 100-meter sprint, 110-meter hurdles, and a serve-return task in tennis. In the volleyball serve-receive task, participants were instructed to receive serve on fifty trials: ten were jump-serves, twenty were float serves to land near the baseline, and twenty were float serves to land away from the baseline. Three probe points were established for the primary task (during the opponent's serve, as the ball carried over the net, and when the participant was about to hit the ball).

The main effects for position of the ball and type of serve were both significant. Float serves showed higher attentional demands than jump serves. Reaction time trends were similar for both serves. At the point of contact by the server, reaction times were fastest. Reaction times were slower as the ball carried over the net and slowest as the player received the ball. This allows us to interpret that float serves require more mental effort and that attentional demands increase over time during a serve-receive task and reach a maximum just prior to contact.

In the 100-meter sprint and 110-meter hurdles, eight expert sprinters and eight expert hurdlers conducted four trials in their specific event on four different days. For the sprint task, four probe points were used (at the start, immediately after coming out of the blocks, 50-meter mark, and at the final thrust to the finish line). In the hurdle task, five probe positions were used (at the start, as the athlete was about to jump over the first 
hurdle, at the 50-meter mark, as the athlete was about to jump over the final hurdle, and at the final thrust to the finish line). Athletes competed against seven other runners to make the primary task (i.e. running as fast as possible) more realistic.

The pattern for reaction times was similar in both groups. At the beginning and end of each race, reaction times were slower than at the intermediate probe points. All reaction times during the race were at least four times higher than control. It seems intuitive that the start and finish of a race would require the greatest mental effort compared to the middle parts of the race. For instance in hurdling, at the start of the race, the runner must be focused on the starting gun, getting out of the starting blocks cleanly, and ensuring appropriate adjustments are made prior to the first hurdle. The experienced hurdler will create a schema for the number of steps between two hurdles. Since each subsequent hurdle is equidistant, after the successful completion of the first hurdle, the need to readjust is minimal and cognitive demands are reduced. At the end of the race, the hurdlers must break from that schema and judge the distance from the finish line as well as being aware of their opponent's positions before they initiate their forward stretch to the finish line.

The final task of this study was a tennis serve-return. Eight professional tennis players conducted forty trials split evenly across two experimental sessions. An acoustic tone was presented at one of four probe points (when the opponent was about to serve, when the ball was at the net, when the ball hits the near court, and when the participant was striking the ball during the return). 
Time points 1 and 2 showed the least mental demand. Probe position 3 showed the slowest reaction times. This is the point when the participant must quickly and accurately assess the spin and speed of the ball in motion, predict its future direction, and make the necessary physical adjustments to make good contact. Probe position 4 was less demanding as the participant had already moved into position and begun the return.

Rose and Christina (1990) extended this line of applied research by examining the distribution of attention during the aiming phase of pistol shooting. Ten novice, ten subelite, and six elite pistol shooters performed sixty shooting trials in two separate experimental sessions. A secondary task required participants to depress a microswitch with their non-shooting hand as quickly as possible in response to an auditory tone delivered randomly at one of six predetermined time points. The six probe positions were $250,1,000,2,500,4,000,6,500$, and 8,000 milliseconds after the shooter had raised the pistol to the "hold" or "ready" position. The tone was delivered five times at each probe position for both sessions.

Reaction time to the tone, time to complete the shot, and shooting score were recorded for each trial. Aborted shot trials and failure to respond were coded as performance errors and those trials were repeated. Across all skill levels, level of attention increased linearly over time as a function of the presentation of the secondary task. The two more experienced shooting groups aborted more trials compared to novice shooters. Elite shooters also failed to respond to the probe more times than novice shooters. More experienced shooters showed a greater narrowing of focus and unwillingness to execute the shooting task outside optimal conditions. 
Prezuhy and Etnier (2001) continued this trend of examining attentional demands of real-world sport skills using the dual-task paradigm and extended the literature by examining the effects of task difficulty on patterns of attentional demands. They observed forty competitive horseshoe pitchers under normal and an experimental "difficult" condition. Both conditions utilized a regulation horseshoe pit; however, the target stake was only six inches high in the "difficult condition" compared to the standard sixteen inches in the "normal condition." For the dual-task paradigm, the primary task was horseshoe performance, measured as the distance to one-tenth of a centimeter, from the stake. The secondary task was a reaction time test to an auditory cue presented at one of three probe positions during the toss. Probe position 1 was as soon as the throwing hand moved in any direction to execute a toss. Probe position 2 was the point during the pitching motion when the throwing hand reached the highest point of the backswing. Probe position 3 was the point just prior to the release of the horseshoe.

After a short warm-up, individual baseline measurements were taken for the primary and secondary tasks. Participants conducted forty trials at each difficulty level. The presentation of a tone at probe positions 1-4 or administering a catch trial was determined at random for each trial with the constraint that there were an equal number of trials for each. Ten of the trials were administered during each probe position and the remaining ten trials were catch trials with no tone presented to keep the participant's attention focus on the primary task.

Reaction times were greatest at the first and third probe position under both levels of difficulty. These results indicate that attentional demands are greatest at the initiation of the toss and just prior to release. This is in line with previous motor learning literature 
that points toward a non-linear pattern of attention. The authors noted that additional research should be conducted to determine if this pattern is observed in other sportspecific skills.

Sibley and Etnier (2004) used the dual-task paradigm to observe the relationship between decision-making and the time course of attentional demands during a volleyball set. Twenty intermediate volleyball players were recruited to complete a series of twohanded overhead volleyball sets under two conditions. The target was a 36-inch diameter hoop that was placed 12 feet from the participant at net height. Subjects were instructed to pass the ball through the hoop that corresponded with the color of ball presented. If the experimenter tossed a ball that was solid white, the target was the hoop in front of the participant. If the ball was striped, the target was the hoop behind the participant. The participant had to quickly recognize the color of the ball and respond accordingly with the appropriate pass.

In the dual-task condition, participants were expected to perform the same primary task while simultaneously performing an auditory reaction time test. A tone was administered at one of four different probe positions of the ball's flight from experimenter to the participant. Probe position 1 was as the ball was tossed to the participant. Probe position 2 was just prior to the apex of the ball's flight path. Probe position 3 was just after the ball reached its apex and began its decent. Probe position 4 was just prior to the participant making contact with the ball. The participant was instructed to verbally respond to the tone as quickly as possible while giving primary focus to the volleyball pass. 
Results indicated that the initial probe position imposed the highest attentional demands on the player. The initial tracking of the ball's flight pattern, choosing the direction of the set, and planning the subsequent motor response happen quickly and require greater cognitive resources for a successful pass to occur. Probe positions 2 and 3 showed the fastest reaction times. This finding is supported with other attention research (Singer et. al., 1998) that found participants can quickly estimate the ball's trajectory and do not need to devote attentional resources to constantly tracking the ball in flight. The final probe position was the second most demanding portion of the ball's flight. At this point, the player must make final adjustments before contact. This requires a higher degree of fine motor control and therefore, increased attentional demands.

In a recent study conducted by Price et al. (2009), a dual task paradigm was used to examine the pattern of attention demands during the closed-skill task of a basketball free throw. An auditory tone was presented at one of four probe positions; (1) Preparation, (2) Pre-shot, (3) Shot, and (4) Flight. A catch probe, where no tone was administered, was also used to reduce anticipatory effects.

After separate single-task baselines for reaction time and foul shot performance, subjects completed forty shots under the dual-task model. Foul shot performance was the primary task and scored as made basket (two points), near miss (one point), and complete miss (zero points). Subjects were asked to concurrently perform an auditory reaction time test administered at randomly selected probe positions during the primary task.

Results indicated that PP1 followed by PP2 required the greatest attentional demands. The remaining two probe positions failed to show significant differences over 
baseline measures. These findings suggest that pre-shot routines may not be as automatic as previously thought. Prior to the execution of the motor skill, attentional reserves may be limited due to increased demand for the collection and interpretation of external information relevant to performing the skill.

\section{Foot Dominance}

Researchers, as well as the general public, have a reasonable understanding of (preferred) hand dominance as a behavioral concept. However, when considering the functional asymmetries of foot dominance, the concept is not as clear-cut. In a summary of the research of preferred footedness, Gabbard and Hart (1996) elaborate on three reasonable arguments on the notion of dominant foot. Most studies have followed the operational definition by Peters (1988) that in bilateral contexts, the mobilizing or manipulating foot is the preferred (dominant) limb. MacNeilage (1991) studied primate neurobiological asymmetries and found that posture in early true primates was necessarily asymmetrical. In a tree habitat, primates needed to grasp the tree with one side and leap or feed with the other side, resulting in the development of both manual and pedal asymmetry. MacNeilage implies that these complementary role characteristics pertain to humans as well and that the operative side is dominant for most persons. Previc (1991) posits that there is no clearly dominant limb in bilateral contexts because one foot must provide postural support while the other executes the voluntary (mobilizing) action. In order for this proposition of complementary role action to hold true, however, neuropsychological demands required for successful completion of each action (mobilization and stabilization) must be equivalent. In kicking a ball, as well as 
most bilateral tasks, the mobilization task will require the greatest neurological demand. For this study, we followed Peters suggestion in that, "the choice of foot for kicking is as compelling as the choice of hand for writing" (p. 183) and, thus in this study, the participant's preferred kicking foot was identified as the dominant foot.

\section{Deautomization of Proceduralized Motor Skills}

Other research on attention in sport has focused on how internal and external focus can impact performance. Masters (1992) proposed a "Deautomization of Skills" hypothesis. This hypothesis suggests that proceduralized tasks, which normally operate under reduced cognitive demands, become "unbundled" when attention is redirected to process the individual components. This results in the diminished performance on a previously well-learned skill. Using the dual-task paradigm, researchers have examined how selectively attending to certain components during performance of a skill can result in performance degradations.

Beilock et. al. (2002) conducted two studies to examine the impact of attention on real-world sport skills. In their first experiment, twenty-one experienced golfers performed a series of golf putts from nine different locations under two conditions. In the skill-focused condition, participants were instructed to concentrate on a specific element of their swing. In the dual-task condition, participants were instructed to simultaneously complete the putting task and verbally respond to prerecorded tones presented at random intervals. Compared to the skill-focused condition, experienced golfers performed significantly better during the dual-task condition. 
The second Beilock et. al. (2002) experiment was intended to extend the findings of the first experiment to include level of skill proficiency and differences in dominant and non-dominant foot performance. Ten novice and ten experienced soccer players participated in a six-cone soccer dribbling task. The skill-focused condition involved participants focusing their attention to the side of the foot that last made contact with the ball. The dual-task condition required participants to dribble through the course and monitor a prerecorded list of words. Presented at random intervals within a list of other words, participants were instructed to verbally respond when they heard the target word, "thorn".

As predicted, regardless of foot, novice soccer players performed better under the skill-focused condition. In the non-dominant foot performance, experienced soccer players also performed better in the skill-focused condition. With their dominant foot, experienced players were able to function better with the word monitoring condition. These results corroborate theories of skill acquisition. In circumstances where proficiency in a particular skill is not well learned, it may be beneficial to direct attention to specific aspects of that skill. As skill improves and becomes proceduralized, attention to specific aspects of the skill may be detrimental to performance.

Ford, Hodges, and Williams (2005) conducted a study to examine the effects of attentional-focus manipulations on expert and novice performance on a soccer dribbling task. Ten skilled and ten less-skilled participants were instructed to dribble a soccer ball through a six-cone, indoor slalom course. Each subject performed the task with their dominant foot under one of four conditions: (1) internal, skill relevant focus $\{$ foot $\}$; (2) internal, skill irrelevant focus $\{\operatorname{arm}\}$; (3) word-monitoring $\{$ word $\}$; and (4) no attentional 
manipulation \{control\}. Skilled participants also performed additional trials under the same conditions with their non-dominant foot.

With the sample of less-skilled performers, attention to the internal, taskirrelevant (hand) and word-monitoring cues impaired performance on the dribbling task compared to control. Attention to the internal, skill relevant cue (foot) did not impair performance. For the skilled performers, both internal-focus conditions hindered performance. There were no significant differences found for skilled performers in the non-dominant foot trials. These results also support the deautomization of skills hypothesis because both internal-focus conditions showed performance declines in experienced athletes. 


\section{CHAPTER III \\ OUTLINE OF PROCEDURES}

\section{Participants}

Participants consisted of fifteen $(\mathrm{N}=15)$ players from local traveling $\mathrm{U}-16, \mathrm{U}-17$, U-18, and U-19 soccer teams and university students with previous competitive soccer experience and currently participating in the sport. Participant ages ranged from 14-22 (mean age of $17.87 \pm 2.39$ years). Each participant's years of soccer playing experience was assessed by self-report. Only same-side dominant (right-foot and right-hand dominant; left-foot and left-hand dominant) participants were selected to participate in this study. Players with previously diagnosed hearing problems were excluded from the study. A self-report question was used to ascertain dominance. All procedures were conducted in accordance with the ethical guidelines of the University of North Carolina at Greensboro.

\section{Measures/Instrumentation}

In order to ensure generalizability, steps were taken to ensure the most accurate "real world" conditions. Testing was conducted based on current FIFA-regulations. Testing occurred on a natural-grass soccer field. A FIFA-regulation soccer goal was placed twelve yards away from the testing station. To maintain consistency while simulating the difficulty in shot placement required during a penalty kick, the goalkeeper 
was replaced with three scoring zones. The goal was reduced to smaller "scoring zones" for scoring purposes. The primary target was the two outer zones measuring inward twoyards from the goal posts. A secondary target goal was the remaining four-yards located between the outer scoring zones (see Figure 1). Target zones were distinguished by 1inch PVC tube spanning the full height of the goal. The participant placed the ball at the penalty spot before each trial. Scoring was based on successful execution of the task with the ball passing through the scoring zone. If the participant was successful, he/she was awarded two points. If the ball entered through the secondary scoring zone, the participant received one point. If the ball failed to cross the goal line through the mouth of the goal, the participant received zero points for that trial. If the ball hit the goal posts or the target zone markers and did not cross the goal line, the participant received zero points for that trial. Participants were instructed to perform the kick as if they were doing so in a game situation.

Soccer is played outdoors with environmental noises as a common occurrence. Auditory capacity should not be particularly challenged in a soccer kick and therefore the use of an auditory probe should not produce any structural interference. A laptop equipped with external speakers and a digital voice recorder were used to deliver the auditory stimuli and record the participant's response. The Olympus WS-400S digital voice recorder and ME-52W noise-cancelling microphone were selected due to their lightweight and portable design. The WS-400S digital voice recorder is 3.70 by 1.50 by 0.40 inches and weighs 1.60 ounces. The ME-52W microphone is 4.40 by 2.00 by 0.80 inches and weighs 0.50 ounces (Olympus, 2009). The ME-52W was selected to ensure optimal uni-directional recording in an outdoor setting. The ME-52W optional tie-clip 
was also used to ensure that the microphone would stay in place while the participant was in motion. The wireless recording devices that were used allowed the participants' unrestricted movement compared to a traditional wired headset/microphone.

Audacity 1.3.7 (Mazzoni, 1989, Boston, MA) is an open source audio editor and recorder. This software was used to record the auditory stimuli as well as the participant's verbal response. The software includes a spectrogram and "plot spectrum" analyzer for detailed frequency analysis. To ensure experimenter accuracy in providing the auditory stimulus at the intended probe point, digital videotaping was also conducted during the pilot testing sessions. Video files were analyzed by an external collaborator.

The tone was administered manually by the experimenter through the Audacity 1.3.7 software. The tone was a computer-generated, 2,000 hertz tone that lasted 0.350 seconds. The participant was instructed to respond to the tone as quickly as possible with a predetermined verbal cue. "BALL" was used as the verbal cue for the dual-task model of this experiment. According to previous research (Beilock et. al., 2002; Ford, Hodges, \& Williams, 2005), attending to component processes during the execution of a proceduralized task can negatively impact performance. However, external taskirrelevant cues have been shown not to interfere with performance of well-learned tasks in experienced athletes (Ford, Hodges, \& Williams, 2005).

\section{Procedures}

Fifteen participants were recruited for participation in this study. Participants first completed a consent form and demographic questionnaire detailing age, previous soccer 
experience, position most frequently played, current medications, current level of fatigue, acute/chronic injuries, and dominant hand/foot. Parental consent and participant assent forms were included for participants under 18 years of age. Only individuals with sameside hand/foot dominance were used for this study.

Baseline performance measures for both tasks were established prior to testing. Participants were put through a standardized dynamic warm-up protocol to minimize risk of musculoskeletal injury during testing. Participants performed ten kicks with their dominant and ten kicks with their non-dominant foot that were scored for performance comparison purposes. Then, without kicking the ball, the participant stood over the ball as if he/she was going to perform the kick. After the participant was given the "ready" signal, he/she was asked to respond to the auditory tone as quickly as possible with the verbal cue, "BALL". The presentation of the auditory tone was presented at random within two seconds after the ready signal was given. Ten VRRT trials were averaged and then used as the baseline for secondary task performance comparisons.

Three probe positions (PP) were used during performance of the soccer kick. Probe position 1 (PP1) was operationalized as the participant's second to last step (taken with their non-kicking foot), probe position 2 (PP2) was the next to last step (taken with their kicking foot), and probe position 3 (PP3) was the final step (taken with their nonkicking, or "plant foot") just prior to making contact with the ball. The participants were instructed that the primary task was to accumulate the highest score possible by kicking the ball through the designated scoring zones. To establish inter-observer reliability and accuracy in administering the probe at the specified position, a second observer examined video footage of each trial for a randomly selected participant during pilot testing. 
Participants were informed that the experimenter would select which foot they were to kick the ball with prior to each trial. Participants completed ten trials at each probe position as well as ten catch trials for a total of forty trials with each kicking foot.

The secondary task was for the participant to respond with the verbal cue, "BALL", as quickly as possible to the audible tone presented. To ensure that the secondary task was challenging enough but not so much as to hinder primary task performance, comparisons were made between kick trial performance and baseline measures of kicking performance. Performance on the primary task must not differ significantly from baseline or results could not be included for that participant.

Catch trials were also included to minimize the participant's ability to anticipate the presentation of an audible tone. A catch trial is when the participant is instructed to perform the primary task and no dual-task stimulus is presented. This reduces participant anticipation effects and maintains focus on the primary task performance, thus ensuring more accurate VRRTs during trials with probe positions. During the catch trials, since no auditory stimulus was presented, VRRT was not measured. Kick performance was recorded and compared to baseline measures. As with the probe trials, comparisons between performance during the dual-task and baseline performance should not differ significantly. 


\section{Data Reduction}

The soccer penalty kick is a simple, closed-skill task familiar to the sport. The player was not given instructions on how to kick the ball. The investigator was able to identify three discrete time points in the kicking motion that were consistently observed in all players. The three probe positions identified were relative to the steps leading up to the kick. In particular, the probe positions occurred with the landing of: (1.) the prekick, non-kicking foot, (2.) the pre-kick, kicking foot, and (3.) the pre-kick, plant foot immediately preceding contact with the ball (see Table 1). A second observer examined each trial for a randomly selected participant during pilot testing to ensure that distinct probe positions were easily identifiable and that the tone was accurately administered. Kick performance scores were compiled for each probe position and catch trial for comparison purposes. Kick performance scores during performance of the dual task were compared to baseline measures for each foot position to ensure that the VRRT task remained the secondary task and that the kick task itself was not too challenging. The time of peak attentional demand was determined to be the probe point at which VRRT was slowest.

Digital voice recordings were collected for each trial and analyzed through Audacity software. Following procedures defined by Price et al. (2009), VRRT was defined as the time from the beginning of the computer generated tone to when the verbal response reaches waveform amplitude of $0.1 \mathrm{~dB}$. Recordings were enlarged and slowed to $20 \%$ of the original speed to provide accurate analysis. The beginning of the auditory tone was determined as the initial burst in waveform activity. The envelope editing tool, native to Audacity software, was used to determine the $0.1 \mathrm{~dB}$ threshold for verbal 
response. The point at which the verbal response waveform first reached the defined threshold was determined to be the conclusion of VRRT. Aural and visual identification was used to determine the start and stop of each VRRT measurement. VRRT was measured with a resolution of 0.0001 seconds.

\section{Data Analysis}

The probe position that requires the greatest attentional demand was determined as the probe point where VRRT was slowest. Using Audacity 1.3.7 software, audio recordings from each trial were used to measure VRRT. Sound files were displayed as waveforms and time between waves was measured. The Audacity software program allows for accurate analysis within 0.0001 seconds. The ten VRRTs for each probe position were averaged and used as the dependant measure. The two independent variables were probe position (three levels - one level for each of the three probe positions) and kicking foot (dominant, non-dominant).

Attention functions like a computer processor. When the demand of attending to two simultaneous tasks exceeds available resources, performance diminishes on the secondary task. To measure how the timing of the auditory stimulus affected both kick performance and VRRT, separate repeated measure ANOVAs were used. According to Prezuhy and Etnier (2001), if performance on the primary task significantly drops, the primary task was too challenging to include a secondary task or participants re-prioritized the primary and secondary task. 
With the VRRT probe technique, it is important that the primary task be given attentional priority. If participants invert the priority level of the primary and secondary tasks, the VRRT becomes the primary task and we cannot test our hypothesis. Presenting the probe points in random order as well as the inclusion of catch probes minimized those methodological concerns (Abernethy, Summers, \& Ford, 1998).

To ensure that the primary task remained the priority, a repeated measures ANOVA was used to compare kick performance scores across conditions (the three probe positions, catch trials, and baseline) for each kicking foot. To examine the time course of attentional demands, VRRT was examined using a two-way, repeated measures ANOVA with kicking foot (DFP, NDFP) and probe position (PP1, PP2, PP3) as the within-subjects independent variables. Significant interaction effects were followed up examining the means at the probe positions (see Figure 3) and by conducting dependent samples t-tests to compare each possible pair of means. 


\section{CHAPTER IV}

\section{RESULTS}

\section{Baseline Performance Trials}

The results from the repeated measures ANOVA showed that there was no significant difference in kick performance for shots taken with the dominant foot as a function of condition, $\mathrm{F}(4,56)=.652, \mathrm{p}>.05$, partial $\eta^{2}=.044$. There was also no significant difference in kick performance for the shots taken with the non-dominant foot as a function of condition, $F(4,56)=1.90, p>.05$, partial $\eta^{2}=.119$. Table 4 shows the means and standard deviations for performance for all conditions.

\section{Experimental Trials}

The results of the 2-way RMANOVA for VRRT indicated that the main effect for probe position was not significant, $\mathrm{F}(2,28)=3.28, \mathrm{p}=0.053$, partial $\eta^{2}=0.19$. The main effect for foot was also not significant, $F(1,14)=0.17, p=0.69$, partial $\eta^{2}=0.01$. However, there was a significant interaction of probe position $\mathrm{x}$ foot, $\mathrm{F}(2,28)=6.09$, $\mathrm{p}=0.006$, partial $\eta^{2}=0.30$. Examination of the significant interaction (see Figure 3) indicated that PP1 had the greatest attentional demands in the DFP condition. In contrast, the greatest attentional demands were seen at PP3 in the NDFP condition. The DFP condition followed the pattern of PP1, PP3, and then PP2. The NDFP condition followed the pattern of PP3, PP1, and then PP2. In both conditions, PP2 was observed to be the least demanding. For VRRT for the NDFP, follow-up tests indicated that PP1 was 
significantly different from PP2, $t(14)=2.30, p<.05$, but that neither PP1 nor PP2 were significantly different from PP3, $p>.05$. In the DFP, follow-up tests indicated that PP1 was significantly different from PP2, $t(14)=3.42, p<.05$, but neither PP1 nor PP2 were significantly different from PP3, $p>.05$. Follow-up tests for comparisons between PP as a function of foot indicated that only PP1 was significantly different between kicking foot, $t(14)=-3.98, p<.05$. Neither PP2 nor PP3 were significantly different as a function of kicking foot, $p>.05$. 


\section{CHAPTER V}

\section{CONCLUSIONS}

The purpose of this study was to examine the time course of attention during a soccer penalty kick and the impact of the use of dominant and non-dominant feet. Because kick performance did not change across probe positions as compared to baseline performance measures, it can be assumed that primary task focus was maintained (Prezuhy \& Etnier, 2001). Given our results on these performance measures, changes in VRRT can be attributed to varying degrees of attentional demand in the dual-task paradigm.

The hypothesis that VRRT would follow the same pattern in the NDFP condition as the DFP condition was partially supported. Based on follow-up tests for the significant interaction between PP as a function of foot, the pattern was the same. In both foot conditions, VRRT was slowest at PP1 and neither PP2 nor PP3 were significantly different from PP1. This is in line with past research as the initial planning phase is considered the most "attention-intensive" and vulnerable to distraction (Price, 2009; Sibley \& Etnier, 2004; Prezuhy \& Etnier, 2001).

However, there were differences in VRRT as a function of kicking foot such that VRRT was slower at PP1 in the DFP condition than the NDFP condition. The higher attentional demands observed at PP1 for the DFP may reflect that the athlete has greater confidence in kicking with this foot and, therefore, pre-plans some aspects of his/her 
performance. Players may determine the location of their shot, the force required for the shot, and the bend of the ball's trajectory. These "higher order" considerations may only be taken into account when players feel comfortable in their own abilities. That is, if players have concerns about their ability to strike the ball with their non-dominant foot, they may allocate their resources on more fundamental aspects of the motor skill and limit thoughts about more challenging components of the kick. If players are comfortable with their ability, they may tap into available resources to execute those higher order tasks and this may be reflected by higher attentional demands earlier in the approach when kicking with their dominant foot.

A second explanation for the slower reaction times at DFP-PP1 focuses primarily on the demands of the non-kicking foot. Aside from actually making contact with the ball, successful kick performance also requires approaching the ball in a manner that results in the plant foot being properly placed and the approach speed being properly judged. Therefore, it also seems logical to assert that increased attentional demand could be a result of task requirements of the non-kicking foot. In the DFP condition, the $2^{\text {nd }}$ to last step on the approach and the final plant foot is made with the non-dominant foot. Thus the slower VRRT shown in PP1 and PP3 could be explained by an increase in attentional demand required to step with the non-dominant foot in a manner to successfully complete the task. In the NDFP condition, the $2^{\text {nd }}$ to last step on the approach and the final plant foot is made with the dominant foot. The slightly slower VRRT at PP3 may still be a result of striking the ball with the non-dominant foot, however, results of NDFP-PP1 (compared to DFP-PP1) could be explained due to less demands required to step and stabilize with the dominant foot. 
The hypothesis that VRRT during NDFP would be slower than DFP was not supported. In considering these results and taking into account the variability in the other proposed measures of foot dominance (see Table 3), it brings up further considerations regarding the attentional demands of stabilizing, striding, planning, and striking the ball. In a kick such as the one used in this study, the second to last step before striking the ball (PP1) is critical in placing the athlete at the proper distance and orientation to the ball. PP3 is also critical as it represents the final opportunity for adjustments prior to making contact with the ball. One way to interpret our results would be that in the DFP condition, the participant was required to balance with his/her non-dominant kicking foot at both PP1 and PP3 (requiring greater attention). Consequently, the higher attentional demands at those points may reflect the increased demands required for stabilizing and planning when stepping with the NDF. In this condition, however, the athlete then struck the ball with the dominant foot (requiring less attention). These overall demands might logically be on balance with the demands of balancing with the dominant foot at PP1 and PP3, and then striking with the NDF. These results may lend credibility to assertions that there is no dominant foot in bilateral contexts (Previc, 1991) because of the requirements of stabilizing and balancing with one foot to strike the ball with the other.

Due to the unique nature of the soccer kick, it is difficult to compare our results to past literature. The probe points for this study preceded those used in other studies relative to the execution of the skill. In the Prezuhy and Etnier (2001) study of horseshoe pitchers, the probe points selected were the start of the backswing, full backswing, and just prior to release. In our study, there was no true "backswing". The kicking leg simply drags through to contact from the last step with the kicking foot. The final probe 
position, PP3, would therefore represent the "full backswing" of the kick. There was no probe for the point just prior to contact with the ball. In the Price et. al. (2009) study of basketball free throw shooting, the probe positions were during the pre-shot routine, the first motion of the shot, just prior to release, and during the ball's flight to the hoop. Comparisons to this study, again, do not make sense as the probes used here do not match.

Before discussing directions for future research, it is important to note its limitations. While attempts were made to maintain high ecological validity, these results cannot generalize to real-world performance. Some planning was involved to kick into the scoring zones, however, that is not the same as the "mental chess match" that occurs between kicker and goalkeeper.

The last concern offered by Abernethy, Summers, and Ford (1998) involves the presentation of attentional fluctuations across time. RT tests typically use probe positions, or defined segments of a specific task. For instance, probe positions for a baseball pitching task could be (A) preparation, or the pitcher's "wind up" phase, (B) initiation, or the player's initial forward movement to just prior to release, and (C) execution, or the phase from release to follow through. It is possible that during the probe positions determined for the kicking task, attentional demands of one task component could have carried over into subsequent phases, biasing the data. This error could be minimized by plotting reaction time relative to response completion. However, given the limitations in current technology, I was unable to do so. 
Future research should be directed at other skills that would fall under the classification of "striking" tasks. Sport skills such as, soccer passing, soccer and football punting, football place kicking, are just a few examples of such tasks with similar and equally identifiable probe points. This would allow for more accurate comparisons in the time course of attention.

Research should also be directed towards the aforementioned complexities in assertions of foot dominance. With a clearer understanding of functional asymmetry and foot dominance, we can then move toward applied research on more effective training techniques for soccer. For instance, if findings ultimately show that balance and stability are responsible for increased attentional demands, perhaps youth training programs should address those skills first.

In summary, the findings of this study generally suggest that the attentional demands of a soccer kick may vary based on unique factors associated with functional asymmetry. Taken in isolation, this might suggest that players should also incorporate some balance training into their practice regimen. The results of this study, however, do make clear the importance of furthering our understanding of the time course of attention in motor skills and foot dominance. 
Table 1: Probe Positions

\begin{tabular}{|c|c|c|}
\hline Probe \# & Probe Name & Probe Description \\
\hline 1 & Preparation & $\begin{array}{l}\text { The second to last step before striking the ball (taken with their } \\
\text { non-kicking foot) }\end{array}$ \\
\hline 2 & Mid-kick & $\begin{array}{l}\text { The next to last step before striking the ball (taken with their } \\
\qquad \text { kicking foot) }\end{array}$ \\
\hline 3 & Pre-contact & $\begin{array}{l}\text { The final step before striking the ball (taken with their non- } \\
\qquad \text { kicking, or "plant foot") }\end{array}$ \\
\hline & Catch & No tone is sounded - Used to minimize anticipatory effects \\
\hline
\end{tabular}


Table 2: Participant Demographics

\begin{tabular}{|c|c|c|c|c|c|c|c|}
\hline Subject & $\begin{array}{c}\text { Age } \\
\text { (years) }\end{array}$ & Gender & $\begin{array}{c}\text { School } \\
\text { Affiliation }\end{array}$ & $\begin{array}{c}\text { School } \\
\text { Experience } \\
\text { (years) }\end{array}$ & $\begin{array}{c}\text { Club } \\
\text { Experience } \\
\text { (years) }\end{array}$ & $\begin{array}{c}\text { Additional } \\
\text { Experience } \\
\text { (years) }\end{array}$ & Position \\
\hline 001 & 19 & Female & College & 8 & 2 & 0 & $\mathrm{D}, \mathrm{M}$ \\
\hline 002 & 19 & Female & College & 6 & 8 & 0 & $\mathrm{~F}, \mathrm{M}$ \\
\hline 003 & 19 & Female & College & 7 & 2 & 0 & $\mathrm{D}, \mathrm{M}$ \\
\hline 004 & 18 & Female & $\begin{array}{l}\text { High } \\
\text { School }\end{array}$ & 10 & 7 & 4 & $D, M$ \\
\hline 005 & 15 & Female & $\begin{array}{l}\text { High } \\
\text { School }\end{array}$ & 3 & 7 & 0 & $M, F$ \\
\hline 006 & 15 & Female & $\begin{array}{l}\text { High } \\
\text { School }\end{array}$ & 1 & 6 & 0 & $M$ \\
\hline 007 & 18 & Male & $\begin{array}{l}\text { High } \\
\text { School }\end{array}$ & 4 & 6 & 3 & D \\
\hline 008 & 21 & Male & College & 4 & 3 & 0 & D \\
\hline 009 & 19 & Female & College & 3 & 0 & 0 & $\mathrm{~F}$ \\
\hline 010 & 15 & Male & $\begin{array}{l}\text { High } \\
\text { School }\end{array}$ & 4 & 6 & 0 & $M, F$ \\
\hline 011 & 22 & Male & College & 6 & 2 & 0 & $M$ \\
\hline 012 & 20 & Male & College & 2 & 0 & 0 & $\mathrm{M}$ \\
\hline 013 & 14 & Male & $\begin{array}{l}\text { High } \\
\text { School }\end{array}$ & 4 & 4 & 2 & $M, F$ \\
\hline 014 & 16 & Female & $\begin{array}{l}\text { High } \\
\text { School }\end{array}$ & 3 & 12 & 3 & $M, F$ \\
\hline 015 & 18 & Male & $\begin{array}{l}\text { High } \\
\text { School }\end{array}$ & 4 & 6 & 3 & $\mathrm{~F}, \mathrm{M}$ \\
\hline
\end{tabular}


Table 3: Participant Dominance Tests

\begin{tabular}{|c|c|c|c|c|c|}
\hline Subject & Kicking Foot & Writing Foot & 1st Step & $\begin{array}{c}\text { One-Leg } \\
\text { Hop }\end{array}$ & $\begin{array}{l}\text { One-Leg } \\
\text { Stability }\end{array}$ \\
\hline 001 & $\mathrm{R}$ & $\mathrm{R}$ & $\mathrm{R}$ & L & $\mathrm{R}$ \\
\hline 002 & $\mathrm{R}$ & $\mathrm{R}$ & $\mathrm{R}$ & $\mathrm{R}$ & $\mathrm{R}$ \\
\hline 003 & $\mathrm{R}$ & $\mathrm{R}$ & $\mathrm{L}$ & L & L \\
\hline 004 & $\mathrm{R}$ & L & $\mathrm{R}$ & L & L \\
\hline 005 & $\mathrm{R}$ & $\mathrm{R}$ & $\mathrm{R}$ & $\mathrm{R}$ & L \\
\hline 006 & $\mathrm{R}$ & $\mathrm{R}$ & L & $\mathrm{R}$ & L \\
\hline 007 & $\mathrm{R}$ & L & L & $\mathrm{R}$ & L \\
\hline 008 & $\mathrm{R}$ & $\mathrm{R}$ & $\mathrm{R}$ & L & L \\
\hline 009 & $\mathrm{R}$ & $\mathrm{R}$ & $\mathrm{R}$ & $\mathrm{R}$ & $\mathrm{R}$ \\
\hline 010 & $\mathrm{R}$ & $\mathrm{R}$ & $\mathrm{L}$ & $\mathrm{R}$ & $\mathrm{R}$ \\
\hline 011 & $\mathrm{R}$ & $\mathrm{R}$ & $\mathrm{R}$ & $\mathrm{R}$ & $\mathrm{R}$ \\
\hline 012 & $\mathrm{R}$ & $\mathrm{R}$ & $\mathrm{R}$ & $\mathrm{R}$ & $\mathrm{R}$ \\
\hline 013 & $\mathrm{R}$ & $\mathrm{R}$ & $\mathrm{R}$ & L & L \\
\hline 014 & $\mathrm{R}$ & $\mathrm{R}$ & $\mathrm{R}$ & $\mathrm{R}$ & $\mathrm{R}$ \\
\hline 015 & $\mathrm{R}$ & $\mathrm{R}$ & L & $\mathrm{R}$ & $\mathrm{R}$ \\
\hline
\end{tabular}

Test Descriptions (from Gabbard \& Hart, 1996):

Kicking Foot Test - preferred kicking foot

Writing Foot Test - preferred foot for writing name in sand

$1^{\text {st }}$ Step Test - reflexive first step when pushed forward from behind

One-leg Hop Test - preferred foot to hop on

One-leg Stability Test - preferred foot to stand on 
Table 4: Influence of Condition on Kick Performance (Possible performance scores $=0-2$ )

\begin{tabular}{|c|c|c|}
\hline Condition & Mean & Std. Deviation \\
\hline NDFP Baseline & 1.26 & 0.29 \\
\hline NDFP PP1 & 1.47 & 0.34 \\
\hline NDFP PP2 & 1.35 & 0.76 \\
\hline NDFP PP3 & 1.26 & 0.75 \\
\hline NDFP Catch & 1.37 & 0.73 \\
\hline DFP Baseline & 1.49 & 0.35 \\
\hline DFP PP1 & 1.45 & 0.74 \\
\hline DFP PP2 & 1.39 & 0.72 \\
\hline DFP PP3 & 1.40 & 0.76 \\
\hline DFP Catch & 1.37 & 0.76 \\
\hline
\end{tabular}


Table 5: Influence of Condition on VRRT

\begin{tabular}{|c|c|c|c|c|}
\hline Condition & Minimum & Maximum & Mean & Std Deviation \\
\hline NDFP PP1 & 0.15184 & 0.56172 & 0.35678 & 0.10247 \\
\hline NDFP PP2 & 0.14626 & 0.52466 & 0.33546 & 0.09460 \\
\hline NDFP PP3 & 0.12010 & 0.61350 & 0.36725 & 0.12313 \\
\hline DFP PP1 & 0.12759 & 0.64968 & 0.38864 & 0.13052 \\
\hline DFP PP2 & 0.11605 & 0.54674 & 0.33140 & 0.10767 \\
\hline Daseline & 0.13944 & 0.48325 & 0.31135 & 0.08595 \\
\hline & & & & \\
\hline
\end{tabular}


Figure 1: Soccer Kick Task Design

\begin{tabular}{|c|c|}
\hline 2-Point & 2-Point \\
\hline Scoring Zone & 1-Point Scoring Zone \\
\hline
\end{tabular}


Figure 2: Influence of Condition on Kick Performance (Means + SD)

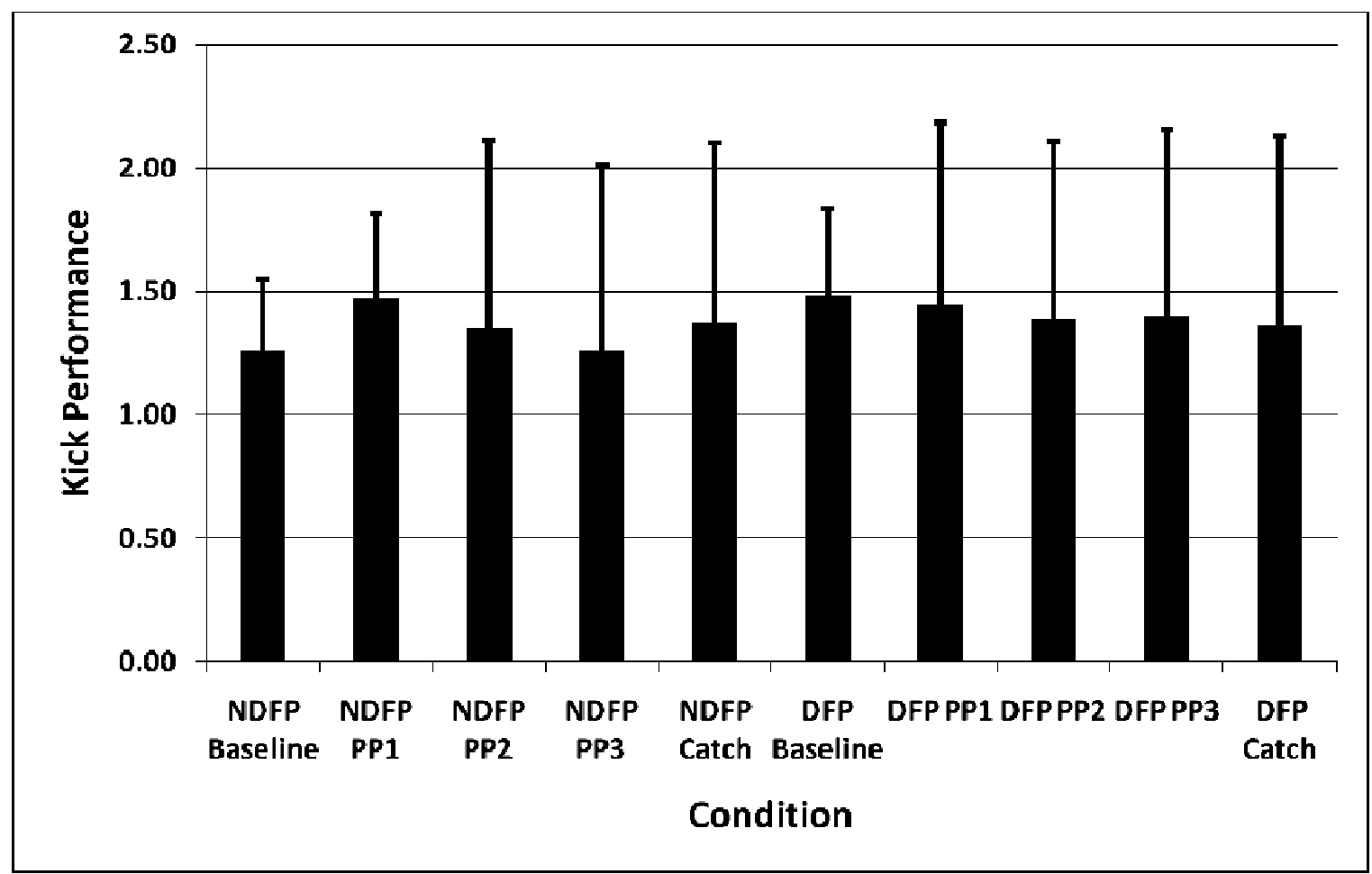


Figure 3: Influence of Condition on VRRT

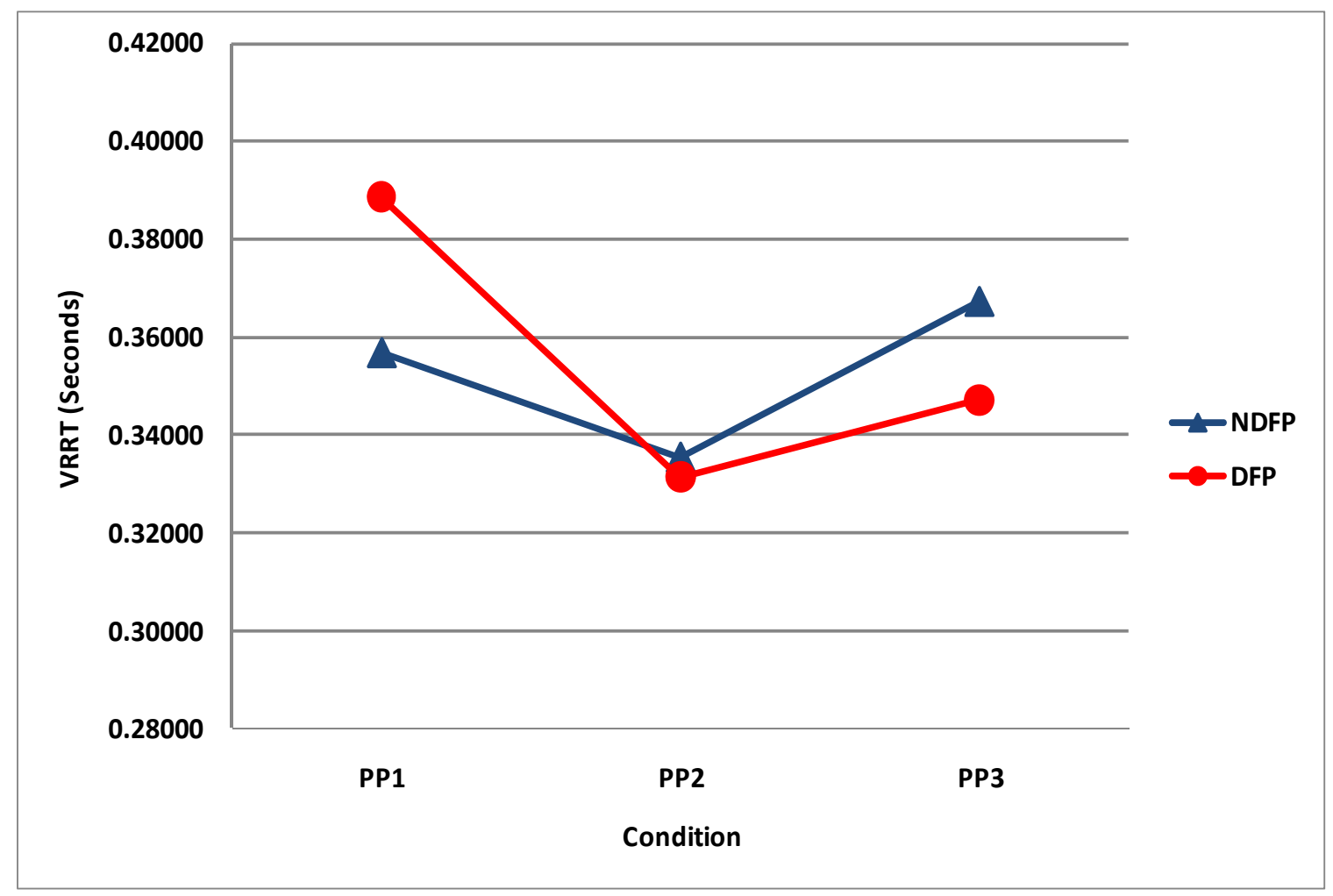




\section{REFERENCES}

Beilock, S. L., Carr, T. H., MacMahon, C., \& Starkes, J. L. (2002). When paying attention becomes counterproductive: Impact of divided versus skill-focused attention on novice and experienced performance of sensorimotor skills. Journal of Experimental Psychology: Applied, 8, 6-16.

Bourke, P. A., Duncan, J., \& Nimmo-Smith, I. (1996). A general factor in dual-task performance decrement. The Quarterly Journal of Experimental Psychology, 49A(3), 525-545.

Castiello, U. \& Umilta, C. (1988). Temporal dimensions of mental effort in different sports. International Journal of Sport Psychology, 19, 199-210.

Gabbard, C. \& Hart, S. (1996). A question of foot dominance. The Journal of General Psychology, 123(4), 289-296.

James, W. (1890). The principles of psychology. Cambridge, MA: Harvard University Press.

Kahneman, D. (1973). Attention and effort. Englewood Cliffs, NJ: Prentice-Hall.

MacNeilage, P. R. (1991). The postural origins theory of primate neurobiological asymmetries. In N. Krasnegor, D. Rumbaugh, M. Studdert-Kennedy, \& R. Schiefelbusch (Eds.), The biological foundations of language development, Hillsdale, NJ: Erlbaum.

Mazzoni, D. (1989). Audacity (Version 1.3.7) [Computer software]. Boston, MA: Free Software Foundation, Inc. Retrieved Jan 13, 2009. Available from http://audacity.sourceforge.net/ 
Peters, M. (1998). Footedness: Asymmetries in foot preference and skill and neuropsychological assessment of foot movement. Psychological Bulletin, 103, 179-192.

Posner, M. I. \& Rossman, E. (1965). Effect of size and location of informational transforms upon short-term retention. Journal of Experimental Psychology, 70, 496-505.

Previc, F. H. (1991). A general theory concerning prenatal origins of cerebral lateralizations in humans. Psychological Review, 98(3) 299-334.

Prezuhy, A. M. \& Etnier, J. L. (2001). Attentional patterns of horseshoe pitchers at two levels of task difficulty. Research Quarterly for Exercise and Sport, 72(3), 293-298.

Price, J. et. al. (2009). Free-Throw Shooting During Dual-Task Performance: Implications for Attentional Demand and Performance. Research Quarterly for Exercise and Sport, 80(4), 718-727.

Rose, D. J., \& Christina, R. W. (1990). Attention demands of precision pistol shooting as a function of skill level. Research Quarterly for Exercise and Sport, 61, 111-113.

Sibley, B. A., \& Etnier, J. L. (2004). Time course of attention and decision making during a volleyball set. Research Quarterly for Exercise and Sport, 75(1), 102-106.

Styles, E. A. (2006). The Psychology of Attention. (2nd 3 ed). Hove, England: Psychology Press. 